\title{
Mixed Convection of Non-Newtonian Fluids through Porous Medium Along a Heated Vertical Flat Plate with Magnetic Field
}

\author{
Ruchi Chaturvedi, Rajesh Kumar, and R. K. Shrivastava
}

\begin{abstract}
In This section we study of Mixed convective heat transfer of non-Newtonian fluids through porous medium with magnetic field on a flat plate has been investigated using a modified power - law viscosity model. This model does not contain physically unrealistic limits of zero or infinite viscosity as are encountered in the boundary - layer formulation with traditional models of viscosity for power - law fluids through porous medium with magnetic field. These unrealistic limits can introduce an irremovable singularity at the leading edge; the present modified model matches well with the measurement of viscosity, and does not introduce irremovable singularities. Therefore, the boundary layer equations can be solved by marching from the leading edge downstream as for Newtonian fluids. The numerical results are presented for a shear-thinning fluid in terms of the velocity and temperature distribution, and for important physical properties, namely the wall shear stress and heat transfer rates.
\end{abstract}

Index Terms-Mixed convection, boundary layer, nonnewtonian, modified power low, finite difference, flat plate, porous medium, magnetic field

\section{INTRODUCTION}

Free convection can have significant effects on forced flows over solid bodies. It can alter the flow field and, hence the heat transfer rate and the wall shear stress. Such effects are particularly enhanced for high-speed rotating machineries due to their large centrifugal forces. The effect of natural convection is accumulative so it cannot be ignored even when the flow acceleration is small. The simplest physical model is a two-dimensional mixed forced and free convection along a flat plate. Understanding of fundamental mechanism of this interaction can help to estimate more accurately the heat transfer rate and pumping power for complex geometries of practical interest in order to prevent unnecessary burn-out of heated surfaces.

Few authors recognize that length scale is associated with the power-law correlation. Due to this length scale, boundary layer problems with power - law, non-Newtonian fluids cannot have simple self-similar solutions. It is nevertheless a common practice to ignore, without justification, the dependence of boundary - layer solutions on the steam wise coordinate. It has been demonstrated in (2008) that such a self - similar solution is actually only

Manuscript received April 19, 2012; revised May 24, 2012

Ruchi Chaturvedi is with AGRA COLLEGE, AGRA (e-mail: ruchiaec3@gmail.com).

Rajesh Kumar is with Mathematics in Eshan College of Engineering Farah Mathura (U.P.) India (e-mail: 79rajesh.ece@gmail.com)

R. K. Shrivastava is with Deptt. of Mathematics in Agra College, Agra (India) valid at the leading edge of the boundary-layer. This similarity solution is the required upstream condition at the leading edge of the flat plate to integrate boundary-layer equations along the stream wise direction.

Traditional power-law correlation, that in the limit of large or small shear rates, Traditional power-law correlations introduce non-removable singularities into boundary-layer formulations for infinite or zero viscosity. Without recognizing the cause of such unrealistic conditions, complex multi-layer structures have been introduced by many authors.

A recently proposed modified power-law correlation is sketched for a number of values of the power index ' $n$ '. It is clear that this new correlation does not contain physically unrealistic limits of zero and infinite viscosities as do traditional power-law correlations. The modified power-law, in fact, fits measured viscosity data better. The constants in the proposed model are fixed with available measurements and described in detail in (2008), where the boundary-layer formulation on a flat plate is described and numerically solved. The associated heat transfer for two different heating conditions is reported (2008). A shear-thinning fluid, whose power-law index is 0.95 , slightly different from Newtonian fluids for which $n=1$, was selected in the study of (2008, 2008). In $(2009,2008$, and 2008) this analysis is extended to fluids whose power-law indexes are $0.6,0.8,1,1.2$ and 1.4 in order to fully demonstrate the effect of non-Newtonian fluids. In this paper, results for mixed convection of nonNewtonian fluids along a vertical flat plate using the modified power-law model for shear - thinning fluid are presented. A similar analysis for natural convection along a vertical heated flat plate appears in (2008). Recently, Molla and Yao (2009) have studied on mixed convection of nonNewtonian fluids along a heated vertical flat plate,

The Nusselt number and shear stress distributions can be well correlated in terms of length scale for all combination of the Reynolds and Rayleigh numbers since the flows are laminar. This is not true for non-Newtonian fluids, since the new length scale, introduced into the formulation by the traditional power-law correlation, becomes the dominant length scale of mixed convection boundary-layers for power-law fluids through porous medium with magnetic field. This will be demonstrated by numerical results presented in section 3 .

\section{FORMULATION OF PROBLEM}

A steady laminar boundary-layer of a non-Newtonian fluid through porous medium along a semi-infinite heated flat plate with magnetic field has been studies. The viscosity 
depends on the shear rate and is correlated by a modified power-law for shear-thinning non-Newtonian fluids. It is assumed that the surface temperature of the plate $T_{w}$, where $T_{w}>T_{\infty}$ Here $T_{\infty}$ ambient temperature of the fluid and $T$ is the temperature of the fluid. The coordinate system is shown in Fig. 1.

Fig. 1 (Physical Model and Coordinates)

The equations governing the flow and heat transfer are

$$
\begin{gathered}
\frac{\partial \bar{u}}{\partial \bar{x}}+\frac{\partial \bar{v}}{\partial \bar{y}}=0 \\
\bar{u} \frac{\partial \bar{u}}{\partial \bar{x}}+\bar{v} \frac{\partial \bar{u}}{\partial \bar{y}}=\frac{\partial}{\partial \bar{y}}\left(v \frac{\partial \bar{u}}{\partial \bar{y}}\right)+g \beta\left(T-T_{\infty}\right) \\
-\frac{\sigma B_{0}^{2}}{\rho} \bar{u}-\frac{v}{K} \bar{u} \\
\bar{u} \frac{\partial T}{\partial \bar{x}}+\bar{v} \frac{\partial T}{\partial \bar{y}}=\alpha \frac{\partial^{2} T}{\partial \bar{y}^{2}}
\end{gathered}
$$

where $\bar{u}, \bar{v}$ are velocity components along the $(\bar{x}, \bar{y})$ axes, $T$ is the temperature, and $\alpha$ is the thermal diffusivity of the fluid. The viscosity is correlated by a modified power-law, which is

$$
v=\frac{k}{\rho}\left|\frac{\partial \bar{u}}{\partial \bar{y}}\right|^{n-1} \text { for } \bar{\gamma}_{1} \leq\left|\frac{\partial \bar{u}}{\partial \bar{y}}\right| \leq \bar{\gamma}_{2}
$$

The constant $\bar{\gamma}_{1}$ and $\bar{\gamma}_{2}$, are threshold shear rates, $\rho$ is the density of the fluid, and $k$ is a dimensional constant, whose dimension depends on the power - law index $n$. the values of these constants can be determined by matching with measurements. Outside of the above range, viscosity is assumed constant; its value can be fixed with data given.

The boundary conditions for the present problem are

$$
\begin{aligned}
& \bar{u}=\bar{v}=0, \quad T=T_{w} \text { at } \bar{y}=0 \\
& \bar{u}=U_{0}, \quad T \rightarrow T_{\infty} \text { as } \bar{y} \rightarrow \infty
\end{aligned}
$$

where $U_{\mathrm{o}}$ is the free steam velocity.

We now introduce the following non - dimensional transformations;

$$
\begin{aligned}
& x=\frac{\bar{x}}{l}, \quad y=\frac{\bar{y}}{l} \mathrm{Re}^{1 / 2}, \quad u=\frac{\bar{u}}{U_{0}}, \quad v=\frac{\bar{v}}{U_{0}} \operatorname{Re}^{1 / 2} \\
& K_{0}=\frac{K U_{0}}{v l}, \quad M=\frac{\sigma B_{0}^{2} l}{\rho U_{0}}, \quad \theta=\frac{T-T_{\infty}}{T_{W}-T_{\infty}}, \quad D=\frac{v}{v_{1}} \\
& \operatorname{Re}=\frac{U_{0} l}{v_{1}}, \quad G r=\frac{g \beta \Delta T l^{3}}{v_{1}^{2}}, \quad \operatorname{Pr}=\frac{v_{1} \rho C p}{k}=\frac{v_{1}}{\alpha}
\end{aligned}
$$

where $v_{1}$ is the reference viscosity, $\theta$ is the dimensionless temperature of the fluid, $R e$ is the Reynolds number and $G r$ is the Grashof number. The length scale is

$$
l=C^{\frac{2}{1-n}}\left[\left(\frac{k}{\rho}\right)^{2} \frac{1}{v_{1}^{n+1}}\right]^{\frac{1}{n-1}} U_{0}^{3}
$$

Substituting variables (2.6a) into Eqs. (2.1) - (2.4) leads to the following non-dimensional equations

$$
\begin{gathered}
\frac{\partial u}{\partial x}+\frac{\partial v}{\partial y}=0 \\
u \frac{\partial u}{\partial x}+v \frac{\partial u}{\partial y}=\frac{\partial}{\partial y}\left[D \frac{\partial u}{\partial y}\right]+\frac{G r}{\operatorname{Re}^{2}} \theta-Q u \\
\frac{\partial \theta}{\partial x}+v \frac{\partial \theta}{\partial y}=\frac{1}{\operatorname{Pr}} \frac{\partial^{2} \theta}{\partial y^{2}}
\end{gathered}
$$

where

$$
\begin{gathered}
D=\frac{k}{\rho v_{1}}\left(\frac{U_{o}}{l}\right)^{n-1}\left|\frac{\partial u}{\partial y}\right|^{n-1}=C\left|\frac{\partial u}{\partial y}\right|^{n-1} \\
Q=\left(M+\frac{1}{K_{0}}\right)
\end{gathered}
$$

where Pr is the Prandtl number. The physical meaning of $\frac{G r}{\operatorname{Re}^{2}}=\frac{1}{U^{2} /(\beta \Delta T)}$ presents the ratio of the length scale that the non-Newtonian effect becomes apparent and the length scale that the natural convection effect grows dominant. For larger $\frac{G r}{\mathrm{Re}^{2}}$, it takes shorter distance for the effect of natural convection becomes dominant.

The boundary conditions $(2.5)$ become

$$
\left.\begin{array}{l}
u=v=0, \quad \theta=1 \text { at } y=0 \\
u \rightarrow 1, \theta \rightarrow 0 \text { as } y \rightarrow \infty
\end{array}\right\}
$$

Next the equations are transformed to parabolic coordinates [1987]

$$
\xi=x, \quad \eta=\frac{\hat{y}}{(2 x)^{1 / 2}}, U=u, \quad V=(2 x)^{1 / 2} v, \theta=\theta(\xi, \eta)
$$

In order to remove the singularity at the leading edge, and to minimize the variation of the boundary-layer thickness for computational convenience. Consequently, Eqs. (2.7) (2.9) become 


$$
\begin{gathered}
(2 \xi) \frac{\partial U}{\partial \xi}-\eta \frac{\partial U}{\partial \eta}+\frac{\partial V}{\partial \eta}=0 \\
(2 \xi) U \frac{\partial U}{\partial \xi}+(V-\eta U) \frac{\partial U}{\partial \eta}=\frac{\partial}{\partial \eta}\left[D \frac{\partial U}{\partial \eta}\right]+2 \xi\left(\frac{G r}{\operatorname{Re}^{2}} \theta-Q U\right) \\
(2 \xi) U \frac{\partial \theta}{\partial \xi}+(V-\eta U) \frac{\partial \theta}{\partial \eta}=\frac{1}{\operatorname{Pr}} \frac{\partial^{2} \theta}{\partial \eta^{2}}
\end{gathered}
$$

The correlation (2.16) is a modified power-law correlation first presented by Yao and Molla (2008), this correlation describes that if the shear rate $|\gamma|$ lies between the threshold shear rates $\gamma_{1}$ and $\gamma_{2}$ then the non-Newtonian viscosity, $D$, varies with the power-law

$$
\mathrm{D}=\left\{\begin{array}{c}
1, \quad|\gamma| \leq \gamma_{1} \\
\left|\frac{\gamma}{\gamma_{1}}\right|^{n-1}, \quad \gamma_{1} \leq|\gamma| \leq \gamma_{2}, \\
\left|\frac{\gamma_{2}}{\gamma_{1}}\right|^{n-1}, \quad|\gamma| \geq \gamma_{2}
\end{array}\right.
$$

of $\gamma$. On the other hand, if the shear rate $|\gamma|$ do not lie within this range, then the non-Newtonian viscosities are different constants as shown. This is property of many measured viscosities.

Eqs. (2.13) - (2.15) can be solved by marching downstream with the upstream condition satisfying the following differential equations.

$$
\begin{aligned}
& -\eta \frac{\partial U}{\partial \eta}+\frac{\partial V}{\partial \eta}=0 \\
& (V-\eta U) \frac{\partial U}{\partial \eta}=\frac{\partial}{\partial \eta}\left[D \frac{\partial U}{\partial \eta}\right] \\
& (V-\eta U) \frac{\partial \theta}{\partial \eta}=\frac{1}{\operatorname{Pr}} \frac{\partial^{2} \theta}{\partial \eta^{2}},
\end{aligned}
$$

Which are the limits of Eqs. (2.17) - (2.19) as $\xi \rightarrow 0$. The corresponding boundary conditions are

$$
\left.\begin{array}{l}
U=V=0, \quad \theta=1 \text { at } \eta=0 \\
U \rightarrow 1, \quad \theta \rightarrow 0 \text { as } \eta \rightarrow \infty
\end{array}\right\}
$$

Eqs. (2.13) - (2.15) and (2.17) - (2.19) are discredited by a central - difference scheme for the diffusion term and a backward-difference a scheme for the convection terms; finally we get a system of implicit tridiagonal algebraic system of equations. The algebraic equations have been solved by a double - sweep technique. In the computation the continuity equation is directly solved for the normal velocity component $V$. hence, the truncation errors are $\mathrm{O}(\Delta \xi)$. The computation is started from $\xi=0.0$ and then marches downstream to $\xi=100$. After several test runs, convergent results are obtained by using $\Delta \xi=2 \times 10^{-9}$ and $\Delta \eta=0.1$ near the leading edge from $\xi=0.0$ to $\xi=10^{-6}$ afterwards $\Delta \xi$ is gradually increased by the relation $\Delta \xi^{(\text {new })}=2 \Delta \xi^{(\text {old })}$ up to $\Delta \xi=0.005$

The physical quantities of principle interest are the wall shear stress in terms of the skin - friction coefficient $C_{f}$ and the rate of the heat transfer in terms of the Nusselt number $\mathrm{Nu}$, which are, respectively,

$$
\begin{gathered}
C_{f}(2 \xi)^{1 / 2}=\left[D \frac{\partial U}{\partial \eta}\right]_{n=0} \\
N u(2 \xi)^{-1 / 2}=-\left(\frac{\partial \theta}{\partial \eta}\right)_{\eta=0}
\end{gathered}
$$

\section{RESULTS AND DISCUSSION:}

Numerical results are presented for the case of a nonNewtonian power-law fluid of shear - thinning $(n=0.6)$ case along with Newtonian fluid $(n=1.0)$ for the value of the Prandtl number $\operatorname{Pr}=1.2$. Computations have been done for threshold shear rates limits of $\gamma_{1}=0.1$ and $\gamma_{2}=10^{5}$ and for two values of the mixed convection parameter $G r / R e^{2}(=0.1$ or 1.0). The non-dimensional viscosity, $D$, given by the modified power-law correlation, which as a function of the non-dimensional shear rate $\gamma$. The singularity experienced at the leading edge for the traditional power-law correlation has been successfully removed without any difficulty by using the present modified power-law correlation. Since the shear stress at the leading edge is inversely proportional to $\sqrt{2 \xi}$ it is infinite there, and $D=\left(\frac{\gamma_{2}}{\gamma_{1}}\right)^{n-1}$ at the leading edge.

The velocity distribution as a function of $\eta$ at selected $\xi$ locations for the power law index $n=0.6$ are depicted in Figures - (5.1) and (5.2) for $G r / R e^{2}=0.1$ and $G r / R e^{2}=1.0$, respectively at $\xi=0$, the velocity distribution is the forced convection similarity velocity profile, from figures - (5.1) and (5.2), it is observed that the natural convection has a significant influence on the flow field. For $G r / R e^{2}=0.1$, it takes longer distance for the natural convection effect becomes dominant. On the other hand, for $G r / R e^{2}=1.0$, the natural convection takes short distance becomes the dominant mode. The velocity distribution is the natural convection has a significant influence on the flow field for magnetic field and porosity. In figure - (5.3), the velocity distribution decreases with increase the values of magnetic field $(M)$. The velocity distribution increases due to increase the value of porosity parameter $(K)$ in figure $-(5.4)$. 
The corresponding temperature distributions are plotted Figures - (5.5) and (5.6), respectively, for different values of $\xi$ and $\operatorname{Pr}$ at $G r / R e^{2}=0.1$, the temperature distribution is larger than any other location since, near $\xi=1$, the temperature distribution is enhanced due the combined mode of forced and natural convection. On the other hand, the temperature distribution decreases as increase in the case of $\xi$, which is expected due to the natural convection mode. In figure - (5.6), the temperature distribution decreases due to increase the values of Pr for natural convection.

The axial distribution of the skin - friction coefficient $C_{f}(2 \xi)^{1 / 2}$ and the Nusselt number $N u(2 \xi)^{-1 / 2}$ are derived in equations - (2.21) and (2.22), respectively, for $\operatorname{Pr}$ $=1.2, n=0.6$ and $G r / R e^{2}=0.1$, with the forced convection limit. It is observed that the forced convection solutions coincide with the full mixed convection solutions for comparison, we have provided only forced convection solutions because the forced convection and the mixed convection length scales are same for the non-Newtonian fluids, but it is difficult to compare with the mixed and free convection solutions due to the different length scales (2008). They clearly show that the free convection effects grow faster for larger $G r / R e^{2}$. The data for the Newtonian fluids $(n=1)$ with shear-thinning non-Newtonian fluid $(n=$ $0.6)$ to contrast the differences of the two fluids. From this comparison it is observed that the skin-friction coefficient decreases and the Nusselt number increases for the shearthinning fluid. Which can correlate $C_{f}(2 \xi)^{1 / 2}$ and $N u(2 \xi)^{-1 / 2}$ into a single curve for all combination of $\mathrm{Gr}$ and $R e$ for Newtonian fluids (1987). But not for nonNewtonian fluids. This indicates that the length scale introduced by the interaction of forced and free convections is not the proper length scale for non-Newtonian fluids. Consequently, we use the length scale associated with the power-law in the current study.

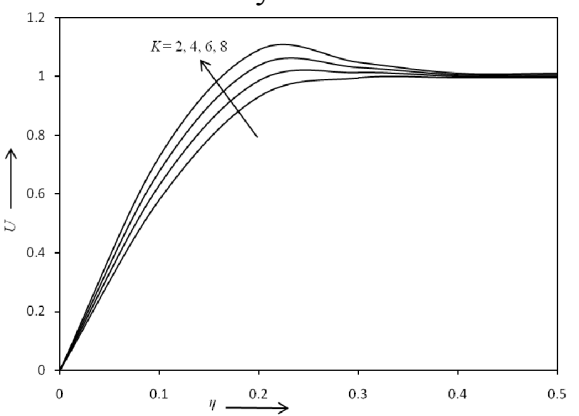

Fig. (5.4). Velocity distribution for different valnes of $\mathrm{k}$ at $\mathrm{Gr} / \operatorname{Re}^{2}=0.1 \& \xi=1$

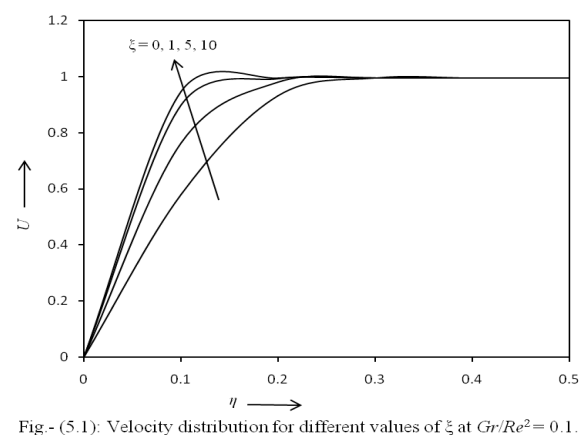

Fig. (5.1). Velocity distribution for different valnes of $\xi$ at $G r / R e 2=0.1$

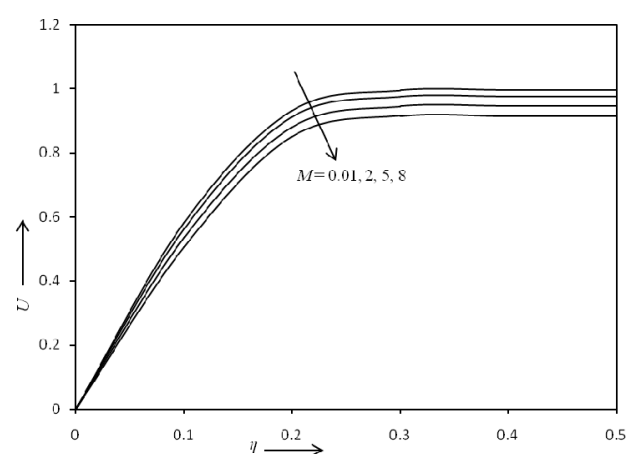

Fig. (5.3). Velocity distribution for different valnes of $M$ at $G r / \operatorname{Re} 2=0.1 \&$ $\xi=1$

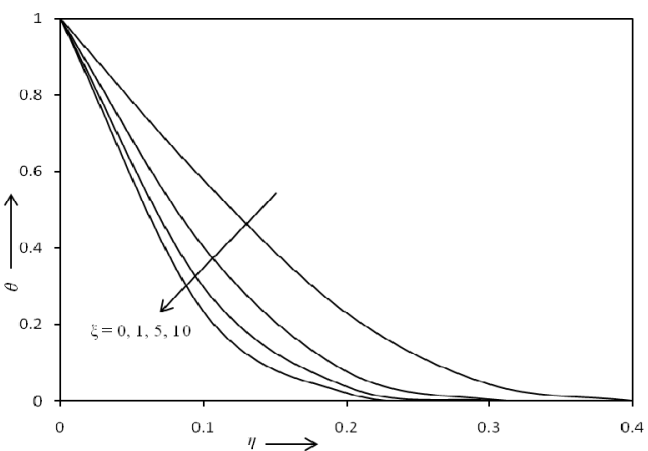

Fig. (5.5). Temperature distribution for different valnes of $\xi$ at $G r / \operatorname{Re} 2=0.1$

\section{CONCLUSIONS}

The proposed modified power-law correlation fits well with the actual measurement of viscosities for nonNewtonian fluids; consequently it does not contain physically unrealistic limits of zero and infinite viscosity introduced into the boundary - layer formulation by the traditional power-law model. The problems associated with the non-removal singularity introduced by the traditional power-law correlations do not exist for the modified powerlaw correlation proposed. This means that the similarity solution exists at the leading edge, which is the natural upstream condition for the non-similar boundary layer problem. Therefore, the proposed modified power-law correlations can be used to investigate other heat transfer problems for shear - thinning or shear thickening nonNewtonian fluids on boundary - layers. The fundamental mechanism that the effect of natural convection eventually becomes dominant when the heating length is long is also properly demonstrated in our computations. For the low heating Case with $G r / R e^{2}=0.1$, the effect of natural convection has not reached its fully developed stage at $=100$

1) The natural convection has a significant influence on the flow field. For $G r / R e^{2}=0.1$, it takes longer distance for the natural convection effect becomes dominant. On the other hand, for $G r / R e^{2}=1.0$, the natural convection takes short distance becomes the dominant mode.

2) The velocity distribution decreases with increase the values of magnetic field $(M)$.

3) The velocity distribution increases due to increase the value of porosity parameter $(K)$. 


\section{REFERENCES}

[1] L. S. Yao and M. M. Molla, "Flow of a non-Newtonian fluids on a flat plate: I boundary layer. J. Thermophys," Heat transfer, vol. 22, no. 4, pp. 758-761, 2008.

[2] M. M. Molla and L. S. Yao, "Flow of non -Newtonian fluids on a flat plate: II Heat Transfer, J. Thermophys," Heat transfer, vol. 22, no. 4, pp. 762-765, 2008.

[3] M. M. Molla and L. S. Yao, "Flow of non -Newtonian fluids on a flat plate a uniform heat flux," ASME, J. Heat transfer, vol. 131, pp. 011702 1-6, 2009.

[4] L. S. Yao and M. M. Molla, "forced convection of non-Newtonian fluids on a heated flat plate," Int. J. Heat mass transfer, vol. 51, pp. 5154-5159, 2008.

[5] M. M. Molla and L. S. Yao, "Natural convection non-Newtonian fluids along a vertical plate plate with a uniform heat flux," $J$. Thermophys. Heat transfer, 2008

[6] S. G. Moulic and L. S. Yao, "Non-Newtonian natural convection along a vertical flat plate with uniform surface temperature," J. Heat transfer, 2008.

[7] M. M. Molla and L. S. Yao, "Mixed convection of non-Newtonian fluids along a heated vertical flat plate," Int. J. heat and mass transfer, vol. 52, pp. 3266-3271, 2009.

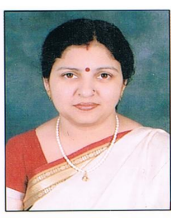

Ruchi Chaturvedi pursuing $\mathrm{PhD}$ from AGRA COLLEGE, AGRA, three Research Papers published in International \& National Journals, Presented two papers in International \& National Conferences. Life member of ISTE, Indian Science Congress, and Authored Two books of Mathematics for undergraduate classes \& two books of junior classes. Member of Editorial Board of ISGBRD. Mail Id:ruchiaec3@gmail.com

Rajesh Kumar Presently Lecturer of Mathematics in Eshan College of Engineering Farah Mathura (U.P.) India Presented papers in 4 international conferences 5 papers are published in International journals Mail Id79rajesh.ece@gmail.com Mob. No.09719163762

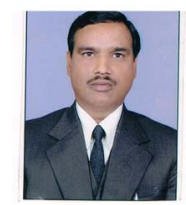

R. K. Shrivastava Presently HOD of Deptt. Of Mathematics in Agra College, Agra (India) 3 students did $\mathrm{PhD}$ under his supervision, 5 students doing $\mathrm{PhD}$ under his supervision, many research papers published in National \& International Journals, presented many papers in conferences. Gave lectures on various topics. Life member of Indian Science Congress \& GANITA, Authored five books for undergraduate classes 